\title{
Escenas de lectura: ¿qué repertorio usan los docentes que enseñan a leer para hablar de su práctica lectora?*
}

\author{
Rosa Cristina Gaete-Moscoso ${ }^{* *}$
}

\section{Resumen}

A partir de una metodología cualitativa narrativa-biográfica, este artículo tiene por objeto ilustrar una noción de lectura, proponiendo una conceptualización de la práctica como ensamble o articulación de elementos que dan forma y orientan la acción. Se identificaron escenas de lectura en el análisis temático de seis biografías de profesores que enseñan a leer. Estos relatos breves mostraban actos o acciones de leer en las cuales los y las entrevistadas "orquestaron" distintos elementos tales como lugares y espacios, personas con sus emociones y sus capacidades, objetos textuales y no textuales, es decir, un repertorio que - expresado como articulación- es interpelado para hablar de la práctica. Se concluye con una discusión acerca del necesario reconocimiento de prácticas diversas.

Palabras clave: historias de lectura, lectura, profesores, repertorio.

\section{Reading scenes: what repertory do teachers who teach to read use to talk about their reading practice? *}

\begin{abstract}
Based on a qualitative narrative-biographical methodology, this article aims to illustrate a notion of reading, proposing a conceptualization of practice as an assembly or an articulation of elements that guides actors' action. Using a thematic analysis of six biographies of teachers who teach to read, scenes of reading were identified. These short stories showed acts or actions to read, in which the interviewees "orchestrated" different elements such as places and spaces, people with their emotions and abilities, textual and non-textual objects, that is, a repertoire that, expressed as articulation is interpellated to talk about the practice. The article concludes with a discussion about the necessary recognition of diverse practices.
\end{abstract}

Keywords: primary teachers, reading, readings stories, repertory.

Recibido: 13/05/2019

Aceptado: 06/08/2019

Este artículo es fruto de la investigación doctoral Historias de lectura de profesores que enseñan a leer. Múltiples prácticas en la narración biográfica y su relación con la enseñanza de la lectura, financiada por el programa Becas Conicyt-Chile, Programa Formación de Capital Humano Avanzado. Una versión de este artículo fue presentada como ponencia en la Conferencia Regional para Latinoamérica y El Caribe IACEP-CDC, “Cognición, inclusión y cultura: desafíos para el aprendizaje en contextos educativos actuales".

* Chilena. Doctora en Sociología, Facultad de Educación de la Universidad Alberto Hurtado, Santiago, Chile.rgaete@uahurtado.cl 


\section{Presentación}

Durante casi dos años, me dediqué a realizar entrevistas biográficas a profesores y profesoras que enseñaban, o habían enseñado, a leer a niños en contexto de escuela. En estas entrevistas los entrevistados narraban su vida y su relación con la lectura. Cada encuentro, cada pedazo de historia, cada reflexión en torno a la lectura me permitía comprender su complejidad y valorar la necesaria reflexión teórica para su estudio y su desarrollo.

Quizás lo que más me impactó de este trabajo fue reconocer cómo las historias de lectura que narraban los docentes apuntaban a experiencias negativas del aprendizaje escolar de esta competencia cultural. Algunas de esas experiencias resultaban, incluso, traumáticas. Engarzado a ello, los docentes tendían a ocultar sus propias prácticas, invisibilizándolas y construyendo una distancia significativa entre la lectura que era narrada en el contexto de la vida cotidiana y aquella que era objeto de enseñanza, tanto la aprendida como la enseñada (Chartier, 2004; Gaete-Moscoso, en prensa; 2017b).

Esta invisibilización — como ha sido discutido por investigadores de la lectura-, correspondería a un mecanismo comparativo que establece el entrevistado con la representación que él tiene y la que cree que tiene su interlocutor acerca de la lectura (Chartier, 2004; Peroni, 2003). Esta invisibilización puede estar reforzada por los modos en que se habla respecto de la lectura, por ejemplo focalizándose solo en el consumo lector (como hace buena parte de la investigación sociológica), o bien, mirando únicamente los componentes cognitivos de ella - como tiende a hacerlo la investigación didáctica一, o incluso realzando solo la "buena" lectura, como pareciera ser el foco en las políticas de fomento lector (Gaete-Moscoso, 2017a).

Aquí solo quiero reforzar la idea de que cualquier persona enfrentada a hablar de su práctica lectora tenderá a discriminar, en primer lugar, lo que considera lectura y, en segundo lugar, determinar si sus prácticas caben en dicha consideración. Si la narrativa social destaca como lectura un corpus escrito o un resultado definido de la actividad (por ejemplo, la comprensión lectora), el individuo tenderá entonces a restringir su percepción de lectura únicamente a ese parámetro (corpus o resultado, o cualquier otro que pueda señalar acá). En parte, esto fue lo que pasó en las narraciones en torno a la lectura 
que las y los docentes entrevistados realizaron. Si la lectura era narrada como una escena más de la vida, adoptaba amplitud, pero si era narrada como un objeto de aprendizaje o como uno de enseñanza entonces se restringía; y si se evaluaba la propia lectura, esta se menospreciaba (Gaete-Moscoso, 2017a).

En un contexto social en el que se cuestionan los modos en que nominamos nuestras realidades, y el lenguaje inclusivo es propuesto como un medio para cambiar las relaciones marcadas por la invisibilización del otro, sostengo que para educadores y cientistas sociales, hacer visibles las prácticas, darles un estatus y un reconocimiento, particularmente a las denominadas prácticas vernáculas (Zavala, 2009), es un paso inicial. Esto implica convocar al diálogo los discursos o narrativas dominantes y contrastarlos con las narrativas cotidianas. Será en el fruto de ese diálogo que podremos avanzar hacia una inclusión basada en el reconocimiento mutuo.

De allí que esta reflexión se articule en torno a dos grandes temas. Por una parte, comprender la noción de lectura a partir de una conceptualización de ella como ensamble o articulación de elementos que operan orquestadamente (Hand \& Shove, 2004; Ingram, Shove \& Watson, 2007; Shove \& Pantzar, 2005), y por otra, utilizando esa conceptualización, problematizar el necesario reconocimiento de la práctica lectora como diversa, infinita y mucho más allá de los cánones preestablecidos, valorándola per se y, por tanto, dando cabida de manera más decidida a formas distintas y nuevas de leer, es decir, de estar en el mundo y de comprenderlo, lo que implica pluralizar el lenguaje que utilizamos cuando nos referimos a ella.

Pretendo, con este ejercicio, ilustrar la complejidad del reconocimiento de las prácticas vernáculas en el espacio escolar y, al mismo tiempo, proponer algunas ideas de cómo, en la enseñanza de la lectura en sus etapas iniciales, podemos hacer este reconocimiento y, por esa vía, imaginar acciones similares para el desarrollo de la comunicación oral y de la producción escrita.

Para alcanzar mi propósito desarrollaré, en primer lugar, los conceptos fundantes de la noción de práctica como ensamble o articulación, señalando cómo se entendería la lectura en esta perspectiva. Luego, en segundo lugar, describiré el trabajo investigativo que nutre esta reflexión, esbozando los resultados obtenidos en la investigación. Por último, abriré una discusión respecto del reconocimiento y diversidad, desde una 
problemática específica ya identificada en la investigación acerca de la lectura (la mencionada “invisibilización” de las prácticas).

\section{La lectura entendida como una orquestación}

Dentro de un encuadre general entre sociología pragmática, de la afectación y teoría del actor-red, la práctica cultural es una actividad social situada (Bernasconi Ramírez, 2013), que integra en su definición y análisis todos los elementos que la hacen posible y la definen. Alejándose de la idea de práctica como una actividad sedimentada en disposiciones aprendidas (Bourdieu, 1997; 2005; Martínez, 2007), en este enfoque se considera la práctica como una entidad viviente y cambiante en la que confluye el individuo con sus capacidades agenciales ${ }^{I}$ (de acción y de reflexión), y el mundo social y material, incluyendo la materialidad de la acción, los objetos, al individuo o agente y los sentidos y significados de la acción.

El estudio de las prácticas tiene como ejemplo el trabajo realizado por el sociólogo francés Antoine Hennion (2002; 2004), quien investigó la práctica de los amateurs de la música contemplando cuatro elementos: el objeto experimentado (la música), el colectivo de aficionados, los dispositivos (discos y aparatos de reproducción de música) y el cuerpo (Barbier y Trepos, 2011). Esta opción analítica complejiza la interpretación y la vuelve circular: la música existe en la música, en lo que los productores (músicos y otros que cumplen la función de producir) producen, en lo que los usuarios o aficionados usan/frecuentan, en los objetos que se materializa, y así sucesivamente.

Las preguntas que surgen en este tipo de análisis se pueden resumir en dos: ¿cómo se hace practicante una persona? Y ¿cómo se articulan los elementos que componen la práctica?

En relación con la primera pregunta, el trabajo de Hennion (2007; 2010) desarrolla una teoría de la afectación (apego o attachement, en francés) que apunta a una relación estrecha entre comunidad de práctica (Gherardi, 2009; Kalman, 2004), materialidades y experiencias.

Término sociológico, se refiere a la noción de agencia, que es la capacidad que tiene cualquier individualidad (persona u otra entidad) para actuar en el mundo, poniendo en juego sus habilidades y competencias. 
En relación con la segunda pregunta, la idea de ensamble se aproxima a la noción de trama, de enlace y de articulación (Ingram et al., 2007) u orquestación (Hand \& Shove, 2004), permitiendo comprender que, además de la diversidad de elementos que entran en juego, estos se organizan y operan de maneras también variadas y múltiples, dando como "resultado" una multiplicidad de prácticas.

El reconocimiento de los variados elementos que componen, intervienen y definen la práctica permite visualizarla como una matriz de acción corporalizada, materialmente mediada y con significados compartidos (Shove \& Pantzar, 2005), de allí que el ensamble y su orquestación no existen fuera de un régimen de sentido de una comunidad y en un tiempo histórico específico.

Así entendida, la lectura sería un ensamble de elementos que se articulan u orquestan entre sí, que existen por la agencialidad del individuo tanto como por la acción proveniente del medio. Utilizando esta herramienta analítica diseñé una investigación en torno a la lectura en la que pudiese ilustrar la práctica lectora o, para ser consistente, las prácticas lectoras de quienes enseñan a leer.

\section{Una investigación usando esta noción de lectura}

\subsection{Respecto del enfoque y el método}

En Historias de lectura de profesores que enseñan a leer (Gaete-Moscoso, 2017a), trabajé desde un enfoque investigativo narrativo-biográfico.

Este enfoque, entendido como "una forma de indagación” (Bernasconi Ramírez, 2011, p. 13) se caracteriza por construir el conocimiento apelando directamente a la voz de los actores y se sustenta en dos supuestos básicos: las personas construyen sentidos sobre sí mismas y el mundo narrándose a sí mismas y narrando lo que les rodea; y la narración es una forma comunicativa fundamental desde el origen de la humanidad (Bernasconi Ramírez, 2011). La práctica narrativa, en tanto práctica social y cultural, produce un objeto (relato) que puede ser tomado, intencionadamente, por la práctica investigativa como un dato, de naturaleza específica, sobre el cual aplicar distintos procedimientos $\mathrm{y}$, en consecuencia, construir significados y comprensiones de los fenómenos sociales, tales como la lectura, identificando las tramas y tópicos movilizados en dicho relato. 
Una forma de narración es la biografía. Este enfoque investigativo y técnica de recolección de datos se caracteriza por interpelar a los actores para que hablen de sí mismos, de su tiempo personal, de su pasado y, en esa ilación, ir explorando un ámbito temático específico (Bertaux, 1980), por ejemplo, la práctica lectora. El gran caudal de literatura que existe acerca de historias, narraciones y relatos de vida ha logrado demostrar que se puede construir un conocimiento a partir del análisis minucioso de partes y/o del todo en la forma de la historia de una persona, de un grupo o de un determinado colectivo. En la naturaleza de este conocimiento, fundamentalmente hermenéutico, destaca la comprensión y la significancia obtenida de él. Se transforma en un lente, una óptica y una puerta para seguir planteándose preguntas y continuar, por esa vía, avanzando en la construcción conceptual (Bertaux, 1980; Bertaux \& Kohli, 1984; Brockmeier, 2000; Cornejo, 2006; Cornejo, Mendoza, y Rojas, 2008; Dorel Bruscas, 2000; Rosenthal, 2004).

Así, entrevisté a un grupo de profesoras y profesores que enseñaban o habían enseñado a leer en los primeros años de escolaridad. En las entrevistas solicité narrar la vida y, en ello, la relación con la lectura. La entrevista, semiestructurada, planteaba preguntas tanto de orden biográfico como profesional. La conducción se caracterizaba por "seguir" el relato como este se iba construyendo y planteando preguntas de precisión cuando era necesario.

El análisis narrativo siguió dos líneas de trabajo: una explícita respecto de lo dicho por el o la entrevistada (análisis temático, Riessman, 2008); y otra referida a los modos en que las narraciones fueron construidas (análisis conversacional, Gubrium \& Holstein, 2000; 2009; Riessman, 2008) tanto en forma como en recursos narrativos interpelados. Este artículo se detendrá en el análisis temático de las historias, en particular, en las escenas de lectura, concepto metodológico que detallaré más adelante.

\subsection{Sobre los participantes}

La decisión acerca de los participantes respondió a tres determinantes de mi trabajo investigativo: preocupación por la formación de profesores (inicial y continua) en didáctica del lenguaje oral y escrito que trabaja a partir de una noción de lenguaje como práctica cultural; la investigación de la lectura (psicológica, sociológica, filosófica y didáctica) ha mostrado una atención preponderante en distintos tipos de lectores, pero muy poca ha 
considerado a los encargados de su enseñanza (salvo para decir lo mal que podrían estar haciéndolo en el aula); y, por último, los ámbitos político y científico tienden a destacar el carácter crítico del estado actual de la lectura el que, aunque cierto, tiende a poner el dedo acusador en los docentes, sin analizar las estrechas relaciones que puede haber entre la invisibilización de su práctica respecto de lo que creen que es la "buena” práctica.

En este contexto, las historias de vida y la relación que tienen los docentes con la lectura permiten problematizar el rol socializador que ellos y ellas cumplen en la escuela (Berger y Luckmann, 1968) y, en particular, en la iniciación a esta práctica socialmente construida que conlleva, como se ha enunciado, más que habilidades cognitivas. Este proceso iniciático a una cultura escrita específica debe estar, sin duda, trasuntado por las propias creencias que se tienen respecto de qué es leer y qué es ser lector (Cisternas y Latorre, 2013; Gaete-Moscoso, en prensa), las cuales son también producto de una trayectoria, de una biografía y de cómo la lectura se articuló o desarticuló en ella.

Se suma a esto la tesis que señala la existencia de una discontinuidad entre una lectura heterogénea (característica de la vida cotidiana, que va en múltiples direcciones, con diversos propósitos y funciones) y una homogénea, esta última característica de la escuela que tiende a restringir la práctica a un determinado corpus y unas funciones específicas (Bombini, 2008; Chartier, 2004; Rockwell, 2001), dando entonces un marco de sentido para aproximarse a la lectura a partir de este dispositivo y, desde allí, construir opciones formativas y didácticas diversas para una práctica dinámica y, en el contexto actual de nuestra sociedad, en pleno proceso de cambio.

En consecuencia, para efectos de este artículo la unidad analítica es la construcción narrativa-biográfica, la lectura en la vida de quienes han sido formados y ejercen su rol enseñando a otros a leer.

Para ello, invité a seis profesores que viven en distintas zonas del país y que, al momento de la indagación, se encontraban desempeñándose en diferentes tipos de establecimientos educativos. Su promedio de edad era de 52 años. Cinco de ellos ejercían en las etapas iniciales de la escolaridad ( $1^{\circ}$ a $4^{\circ}$ año básico) y uno en formación universitaria (específicamente en formación docente de profesores de Educación General Básica, EGB). La mayoría de las participantes fueron mujeres (cinco), lo que es representativo del universo de docentes que trabajan en EGB. La Tabla 1 resume la información en relación con los participantes del estudio. 
Tabla 1. Participantes de la investigación².

\begin{tabular}{|l|c|l|l|l|l|c|}
\hline $\begin{array}{l}\text { Mujer } / \\
\text { Hombre }\end{array}$ & Edad & $\begin{array}{l}\text { Nivel en que } \\
\text { se desempeña }\end{array}$ & $\begin{array}{l}\text { Tipo de } \\
\text { establecimiento }\end{array}$ & Región & $\begin{array}{l}\text { Años de } \\
\text { ejercicio } \\
\text { profesional }\end{array}$ & $\begin{array}{l}\text { Tiempo } \\
\text { de } \\
\text { entrevista }\end{array}$ \\
\hline Mujer & 55 & $2^{\circ}$ año básico & Particular pagado & X & 30 & 284 \\
\hline Mujer & 48 & $1^{\circ}$ año básico & $\begin{array}{l}\text { Particular } \\
\text { subvencionado }\end{array}$ & RM & 27 & 230 \\
\hline Hombre & 63 & Universitario & Particular pagado & RM & 30 & 174 \\
\hline Mujer & 42 & $1^{\circ}$ año básico & $\begin{array}{l}\text { Particular } \\
\text { subvencionado }\end{array}$ & VI & 20 & 163 \\
\hline Mujer & 51 & $\begin{array}{l}1^{\circ} \text { a } 8^{\circ} \text { año } \\
\text { básico }\end{array}$ & $\begin{array}{l}\text { Particular } \\
\text { subvencionado }\end{array}$ & VII & 12 & 295 \\
\hline Mujer & 53 & $1^{\circ}$ año básico & Municipalizado & V & 15 & 325 \\
\hline
\end{tabular}

Fuente: Elaboración propia.

\subsection{Las escenas de la lectura en la vida}

El ejercicio retrospectivo realizado junto con los docentes me permitió identificar los temas que desarrollaban a propósito de su relación con la lectura, y cómo se iban entramando ideas más complejas de lo que significa leer y ser lector. Hubo dos procedimientos de trabajo en relación con las temáticas identificadas: la historia global con su trama (o plot) (Czarniawska, 1998; Gubrium \& Holstein, 2009; Smith \& Sparkes, 2008) y las escenas aisladas, en particular las de lectura, donde centraré la atención.

Entiendo una escena de lectura como un relato acotado en donde aparece un acto o actividad de leer. La escena, aunque parte de una narración de más largo alcance, es reconstruida y aislada del relato mayor para ser abordada en su contenido específico; es una escena porque compone la práctica. Hay una acción o una actividad, una materialidad (un lugar, objetos textuales y no textuales) y unos actores (los que realizan la acción principal y las anexas, y que ponen de manifiesto sus competencias y sus creencias), los que son puestos en relación de distintas maneras por parte de quien relata la historia.

Gracias a la identificación de estas escenas, se construyó un repertorio de la lectura, una suerte de inventario de conceptos (Silber, 2003), sentidos y otros elementos que hacen existir la práctica en la

2 Se mantiene la nomenclatura administrativa chilena vigente al momento de realizar las entrevistas (2013-2014): establecimiento educacional particular pagado, particular subvencionado y municipalizado. 
narración entendidos dentro de una red. Estos componentes se enlazan e interactúan entre sí, dando vida y sentido al acto con toda la complejidad que este tiene. De allí que el repertorio es considerado una performance (Taylor, 2015) narrada de la práctica.

En este enfoque la práctica es lo que la persona hace con el objeto textual en un momento determinado, incluyendo la decodificación y comprensión del mismo. Ese momento tiene a su vez una determinación biográfica $^{3}$ y una histórica ${ }^{4}$ y es posible su existencia en la medida en que existen también los objetos y las condiciones materiales. Las escenas muestran sentidos, funciones y propósitos los cuales operan en relación con los otros elementos. Por último, las escenas muestran las comunidades en donde la lectura surge y/o se inserta. Veamos algunos ejemplos de cómo opera ello en función de los relatos de los participantes del estudio.

\subsubsection{Si no leo, me muero}

Andrea contaba cómo cada noche se sentaban frente a frente junto a su esposo. Estaban recién casados y habían tomado la decisión de ir a trabajar a una isla lejana. No llevaron nada consigo, salvo sus efectos personales básicos y sus libros. Como no tenían energía eléctrica, así como tampoco otros servicios básicos, su momento especial del día era encontrarse cada noche junto al fuego de la cocina y cada uno leía un extracto (un poema, un cuento o lo que quisieran mostrarse uno al otro) como una forma de regalo hacia su pareja. Repitieron esa rutina durante 10 largos años. Andrea y su esposo alimentaron su relación de pareja, desarrollaron sus habilidades de educadores y de colonizadores de un mundo salvaje y sobrevivieron a la ausencia de modernidad gracias a esos textos, como ella los llama: "sus tesoros". Sin esas lecturas, no habrían logrado sobrevivir todos esos años en esa isla lejana, lo que traducido a su propia connotación significa que hubieran muerto sin leer.

\subsubsection{Si leo, me muero}

En un registro completamente distinto, Bernarda narraba el momento en que debió leer el proceso judicial que relataba los hechos del asesinato de su hermano. El asesinato tuvo lugar durante la dictadura militar y fue 
ocultado bajo una versión de enfrentamiento armado. Se preguntó por largo tiempo qué había pasado, cómo la dictadura le había arrebatado a su hermano y por qué les había sucedido aquello. Cuando su hermano murió, ella pensó que no era cierto, que era un error. Pero la desgracia era cierta y el dolor la acompañaría para siempre. Necesitaba saber, necesitaba conocer toda la verdad y, cuando por fin el proceso judicial de los verdaderos acontecimientos se inauguró, tuvo su oportunidad y no dudó en tomarla. Y así lo leyó todo: el proceso inventado y el verdadero; el primero fue redactado inculpando a su hermano y describía cómo lo acribillaron y todo el montaje preparado para hacer parecer la verdad de otro modo. La muerte se le acercó en las palabras y las fotografías allí puestas. Los detalles se le grabaron para siempre y con estos ella sintió que una parte suya moría definitivamente. Bernarda lloró y contó cómo el disparo final entró por una de las órbitas oculares y, recorriendo en diagonal todo el cuerpo, fue a salir por la pelvis; él estaba de rodillas, le pusieron un arma en su mano derecha siendo que él era zurdo. Bernarda lee y en su lectura su hermano muere con ella.

\subsubsection{Platero y yo}

Deysi cuenta que una tarde de otoño estaba sentada junto a su papá en el patio trasero de su casa. Él leía Platero y yo mientras ella lo escuchaba. Antes de entrar en los detalles Deysi señala: "no había profesores que nos motivaran, no debe haberme gustado leer”. ¿Cómo concluye aquello? Porque su papá solo tenía $4^{\circ}$ año básico y, por su actual experiencia profesional, Deysi sabe cómo leen en voz alta los estudiantes de esa edad, es decir, con una lectura entrecortada y con poca entonación. Entonces probablemente su papá leía así y, por tanto, supone que no debe haber sido tan motivador ni suficientemente interesante escucharlo. Sin embargo, Deysi no recuerda concretamente esa "mala lectura", más bien recuerda el sol de la tarde, el patio exactamente a cómo es hoy (con un poco de tierra, con maderas y otros materiales, con camiones estacionados), recuerda la historia de Platero (el burrito que muere en el despeñadero) y de la pena que le provocaba tan triste relato.

\subsubsection{La complicidad de la lectura}

Gabriela salía al mercado todos los sábados junto a sus hermanos y su papá para hacer las compras de la semana. La turba de chiquillos adolescentes que conformaba el cortejo se dividía en distintas tareas y una de 
ellas era buscar y comprar novelas para su padre. Como él debía encargarse de ir por los puestos del mercado pagando las compras de ese pequeño batallón de hermanos y de dos primos allegados, le pedía a cualquiera de ellos que revisara en los puestitos si había algo nuevo para leer. Gabriela no sabe cómo ni en qué momento, los o las encargadas de la revisión y compra de la novela semanal se toparon con las novelas pornográficas y las compraron a escondidas. Así comenzó el tráfico de esos libros que, de mano en mano entre los hermanos y los primos, nutrían lecturas escondidas, cómplices, fuera del ojo censor del papá.

\subsubsection{Performatividad en un papelito}

El día que Carlos recibió su primer sueldo, por primera vez en su vida tuvo en sus manos un cheque. Ese papelito escrito con una cifra en escudos y su nombre completo le indicaba algo, algo que lo diferenciaba de los demás de su familia. Su padre nunca recibió un cheque, ni antes de ese evento ni después tampoco. Un mandato para recibir dinero, una marca de estatus de trabajador, un signo de pertenencia a un mundo escrito distinto del de las novelas y de las historias que Carlos leía hasta ese entonces. Cuando él llegó a su casa con ese objeto, tan poderoso y tan insignificante al mismo tiempo toda la familia, la tribu, se congregó ante él para observarlo, analizarlo, admirarlo, temerle. Cada uno por turno tomaba el papelito, leía el nombre completo de Carlos y se maravillaba.

\subsection{Las escenas de la lectura en la escuela}

Las cinco escenas presentadas contrastaron con otros relatos, situados en el contexto escolar o en el aprendizaje de la lectura en la escuela. En ellos, se ensamblaron elementos de orden similar, sin embargo, eran menos variados ( $\sin$ tiempo, aparición solo de textos específicos para la “instrucción lectora”), eran connotados negativamente (emocionalidades negativas de dolor, vergüenza o miedo ante el error en el aprendizaje de la lectura) o las relaciones entre ellos tendían a la rigidez (las prácticas de lectura no nutren relaciones de apego).

Así, en estas otras escenas la lectura pareciera no tener temporalidad (por ejemplo, en las escenas en la vida el lugar que ocupa el final del día o el momento biográfico en el que sucede una determinada actividad de lectura). Tampoco se apela a ningún propósito específico (como saber la verdad) ni ilustra formas de resolución frente a la vida (complicidad para 
enfrentar el control parental), no hay relación de apego con una comunidad en particular (por ejemplo, la bandada de adolescentes leyendo pornografía, o la vida en pareja). Pareciera como si en los relatos de los docentes la lectura se desnaturalizara cuando sucede en la escuela y, lo más complejo, hiciera desaparecer todo el acumulado o aprendizaje previo que ellos y ellas mismas movilizan cuando la lectura sucede en la vida.

\section{Una discusión a continuar}

Si bien cada escena es una composición única podemos identificar los elementos que son puestos en relación en cada una de ellas.

Todas las escenas movilizan emocionalidades. Para quienes hemos estudiado los discursos que se hacen acerca de la lectura, sabemos que la mayoría de los investigadores relevan emocionalidades positivas al referirse a esta práctica. Sin embargo vemos someramente un conjunto variado de emociones y sentimientos vinculados con la práctica: amor, dolor, melancolía, excitación ante lo prohibido y la novedad. La emocionalidad no existe fuera del acto, y el acto no existe sin la red de personas que se sitúan en la escena. En ellas están las parejas, los padres, la fratría. Y cuando el acto es realizado en solitario (la lectura del proceso judicial, por ejemplo) está presente la sociedad, la legalidad.

De este modo, la presencia de personas (concretas o abstractas) implica que en la escena se trasuntan habilidades: seleccionar un fragmento, descodificar (el ejemplo de la lectura en el patio), comprender, complotar, planificar el momento para leer. La puesta en juego de estas habilidades sucede en un tiempo biográfico (el primer sueldo, la escolaridad, el duelo, estar recién casados, la adolescencia) y un tiempo histórico (la dictadura, la época en que no había electricidad en todas partes, el tiempo en que recibir un cheque era excepcional, cuando la alfabetización inicial era precaria, cuando las familias eran grandes). Las habilidades y el tiempo se coordinan en función de un texto específico (la literatura en general, la poesía en particular, el archivo judicial, la novela pornográfica, el cheque).

\section{1. ¿A qué conclusión podemos llegar?}

Si bien hablamos de "la" lectura, el consenso teórico ha destacado su carácter múltiple, cuestión que no necesariamente ha trasuntado ni el 
discurso oficial ni tampoco la representación que solemos tener acerca de esta práctica. Las escenas de lectura ilustran esa multiplicidad y heterogeneidad y en ellas se plasman articulaciones variadas entre sí. La composición no consta solamente de los elementos, sino también de las relaciones que se entablan entre ellos, las cuales están tejidas por un régimen de sentido que precede la escena (por ejemplo, el deber de leer como régimen de sentido es distinto en Andrea - que lo vive como parte de su identidad - y en Bernarda, que lo vive como una obligación para encontrar la verdad), que la moldea y que de seguro la excede (esta idea solo podría argumentarla haciendo alusión a las historias completas).

En las escenas los lectores están comprometidos en la tarea porque la tarea existe dentro del mencionado régimen de sentido, en el cual no solo está la historia previa de la persona, sino la experiencia del momento que le toca vivir. El compromiso de los lectores no aparece centrado en el texto mismo, sino en la relación con "alguien" que, para él o ella, resulta significativo.

Así, esta orquestación tiene lugar cuando los docentes hablan de sus vidas: su experiencia matrimonial, el compromiso político, los primeros pasos en el espacio laboral, la relación con el padre, la vida familiar extendida.

Sin embargo, el reconocimiento de esta diversidad que está dada tanto por los elementos componentes como por la articulación u orquestación existente entre ellos, desaparece cuando se vuelve a narrarla en el espacio escolar. Lo más complejo de esta afirmación es que pareciera que todas estas escenas desaparecen del discurso y dejan de ser interpeladas en la construcción del repertorio, esta vez en la escuela.

No he podido ilustrar más claramente la invisibilización de estas prácticas en la narración que las y los docentes hacen cuando se refieren a ella como un objeto de enseñanza. Es esto una limitante de mi reflexión. Sin embargo, el lector podrá hacerse a la idea que lo que aparece en las narraciones de la lectura realizada en el contexto de la vida transmite una riqueza y una variedad que queda ausente cuando hablan de sus propias experiencias de aprendizaje escolar, o bien, cuando se refieren a su quehacer como enseñantes de ella. El efecto de la escuela en la minimización de la práctica lectora resulta contradictorio en un mundo donde queremos construir una diversidad y aceptación de ella en todo ámbito. Los cambios lingüísticos que se difunden en la cotidianeidad no van aparejados del reconocimiento de una práctica diversa. Este 
reconocimiento implica otorgarle, a todas las lecturas, un estatus, un lugar de evidenciación y una valoración.

De esta reflexión surge una propuesta para la formación inicial docente, que permita diseñar actividades educativas en las que dichas experiencias sean valorizadas como parte de comunidades de práctica (Kalman, 2004; Lerner, 2001) reconocidas como potenciales recursos didácticos (Gaete-Moscoso, 2017b) y que, en una primera instancia, sean abordadas desde una perspectiva metacognitiva y culturalista del conocimiento previo, de modo que la cultura escrita de los docentes, tal como ha sido vivida, tenga un espacio de reconocimiento en el quehacer de la enseñanza, fortaleciendo la identidad del docente como mediador cultural (Munita, 2014a; 2014b).

Esta reflexión también interpela la didáctica del lenguaje, la cual habiendo reconocido explícitamente la existencia de la multiplicidad de maneras de leer, de sus funciones y de los textos posibles de utilizar (Alliende y Condemarín, 2006; Condemarín y Medina, 2000; Galdames, 2009; Solé, 2009) no se ha logrado plasmar en un discurso del reconocimiento, ni en una práctica acorde con ello, tarea que ciertamente excede a la didáctica en sí misma, ya que aquí incide también el desarrollo de la política y otros aspectos de la vida social que no detallaré en este espacio.

En síntesis, este trabajo analítico refuerza la idea de práctica lectora más allá del acto mismo de descodificar un texto o, incluso en una noción ampliada, de comprenderlo. La práctica es experiencia y acto, que existe en y gracias a un espacio temporal, material y psicológico específico: implica habilidades y competencias, moviliza emocionalidades y, por cierto, redunda en consecuencias tanto puntuales como permanentes en el tiempo para el protagonista de la narración. La práctica tiene una historia que precede al individuo y cuando se engarza en su vida, ya sea positiva o negativamente, sigue un curso propio.

Esta forma, de comprender las prácticas lingüísticas implica trabajar el reconocimiento de ellas, por lo que el quehacer docente se ve interpelado a hacer viva la sala de clases trayendo el mundo real y cuestionándolo desde allí. 


\section{Referencias bibliográficas}

Alliende, F. y Condemarín, M. (2006). La lectura: teoría, evaluación y desarrollo. Santiago de Chile: Andrés Bello.

Barbier, R. y Trepos, J. Y. (2011). Humanos y no-humanos: un balance de la etapa alcanzada en la sociología de los colectivos. Revista Trilogía, 3(5), 123-138. https://doi.org/10.22430/21457778.459

Berger, P. L. y Luckmann, T. (1968). La construcción social de la realidad. Buenos Aires: Amorrortu Editores.

Bernasconi Ramírez, O. (2011). Aproximación narrativa al estudio de fenómenos sociales: principales líneas de desarrollo. Acta Sociológica, 1(56). Recuperado de https://doi.org/10.22201/ fcpys.24484938e.2011.56.28611

Bernasconi Ramírez, O. (2013). Hacia una sociología post-antropocéntrica, relacional y pragmática para el estudio del individuo. Teoría social contemporánea. Presentado en XXIX Congreso de la asociación latinoamericana de sociología, Santiago de Chile. Recuperado de http://www.actacientifica.servicioit.cl/

Bertaux, D. (1980). L'approche biographique: Sa validité méthodologique, ses potentialités. Cahiers internationaux de Sociologie, 69, 197225. Recuperado de https://www.jstor.org/stable/40690465

Bertaux, D. \& Kohli, M. (1984). The life story approach: A continental view. Annual Review of Sociology, 10(1), 215-237. https://doi. org/10.1146/annurev.so.10.080184.001243

Bombini, G. (2008). La lectura como política educativa. Revista iberoamericana de educación, 46, 19-36. Recuperado de http:// www.redalyc.org/pdf/800/80004603.pdf

Bourdieu, P. (1997). Razones prácticas sobre la teoría de la acción. Barcelona: Anagrama.

Bourdieu, P. (2005). Capital cultural, escuela y espacio social. México D.F., Madrid: Siglo XXI.

Brockmeier, J. (2000). Autobiographical time. Narrative inquiry, 1o(1), 51-73. https://doi.org/10.1075/ni.10.1.03bro

Chartier, A. M. (2004). La memoria y el olvido, o cómo leen los jóvenes profesores. En B. Lahire (Ed.), Sociología de la lectura (pp. 109137). Barcelona: Gedisa. 
Cisternas, T. y Latorre, M. (2013). ¿Qué conocimientos tienen los profesores sobre la enseñanza y el aprendizaje de la comprensión lectora? Diferentes enfoques para una misma situación. Cuaderno de Educación. Facultad de Educación Universidad Alberto Hurtado. Santiago de Chile, 53. Recuperado de http://biblioteca.uahurtado.cl/ujah/Reduc/ pdf/pdf/txt1023.pdf

Condemarín, M. y Medina, A. (2000). Evaluación auténtica de los aprendizajes: un medio para mejorar las competencias en lenguaje y comunicación. Santiago de Chile: Andrés Bello.

Cornejo, M. (2006). El enfoque biográfico: trayectorias, desarrollos teóricos y perspectivas. Psykhe (Santiago), 15(1), 95-106. https://doi.org/10.4067/s0718-22282006000100008

Cornejo, M., Mendoza, F., y Rojas, R. C. (2008). La investigación con relatos de vida: pistas y opciones del diseño metodológico. Psykhe (Santiago), 17(1), 29-39. https://doi.org/10.4067/ s0718-22282008000100004

Czarniawska, B. (1998). A narrative approach to organization studies. Thousand Oaks, London, New Delhi: Sage Publications, Inc.

Dorel Bruscas, R. (2000). Historias de vida. Homenaje a Rafael Andolz. Estudios sobre la cultura popular, tradición y la lengua en Aragón, 399-428. Recuperado de https://dialnet.unirioja.es/ servlet/libro?codigo=273184

Gaete-Moscoso, R. (en prensa). La identidad lectora del docente que enseña a leer: hacia la consideración de las historias de lectura como insumos didácticos para la enseñanza. Specificités.

Gaete-Moscoso, R. (2017a). Historias de lectura de profesores que enseñan a leer. Múltiples prácticas en la narración biográfica y su relación con la enseñanza de la lectura. (Tesis de doctorado inédita). Universidad Alberto Hurtado, Santiago de Chile.

Gaete-Moscoso, R. (agosto, 2017b). La narración biográfica de la lectura: reflexión sobre esta como recurso didáctico en su enseñanza y su aprendizaje. Presentado en Seminario Lectura e Inclusión, Santiago de Chile.

Galdames, V. (2009). Desarrollo de la comprensión lectora: ¿un desafío pendiente o un concepto en permanente evolución? Cuaderno de Educación. Facultad de Educación Universidad Alberto 
Hurtado. Santiago de Chile., 12. Recuperado de http://repositorio.uahurtado.cl/bitstream/handle/11242/6552/p 3 . pdf?sequence $=1$

Gherardi, S. (2009). Practice? It's a matter of taste! Management Learning, 40(5), 535-550. https://doi.org/10.1177/1350507609340812

Gubrium, J. F. \& Holstein, J. A. (2000). Analyzing interpretive practice. En N. K. Denzin \& Y. S. Lincoln (Eds.), Handbook of qualitative research. Second edition. (pp. 487-508). Thousand Oaks: Sage Publications, Inc.

Gubrium, J. F. \& Holstein, J. A. (2009). Analyzing narrative reality. Thousand Oaks: Sage Publications, Inc.

Hand, M. \& Shove, E. (2004). Orchestrating concepts: Kitchen dynamics and regime change in good Housekeeping and Ideal Home, 1922-2002. Home cultures, 1(3), 235-256. https://doi. org/10.2752/174063104778053464

Hennion, A. (2002). La pasión musical. Barcelona, Buenos Aires, México D.F.: Paidós.

Hennion, A. (2004). Une sociologie des attachements. D'une sociologie de la culture à une pragmatique de l'amateur. Pratiques musicales, 85(3), 9-24. https://doi.org/10.3917/soc.085.0009

Hennion, A. (2007). Those things that hold us together: Taste and sociology. Cultural sociology, 1(1), 97-114. https://doi. org/10.1177/1749975507073923

Hennion, A. (2010). Gustos musicales: de una sociología de la mediación a una pragmática del gusto. Comunicar, $17(34), 25-33$. https:// doi.org/10.3916/c34-2010-02-02

Ingram, J., Shove, E., \& Watson, M. (2007). Products and practices: Selected concepts from sciences and technology studies and from social theories of consumption and practice. Design Issues, 23(2), 3-16. https://doi.org/10.1162/desi.2007.23.2.3

Kalman, J. (2004). El estudio de la comunidad como un espacio para leer y escribir. Revista Brasileira de Educação, 26. https://doi. org/10.1590/s1413-24782004000200002

Lerner, D. (2001). Leer y escribir en la escuela: lo real, lo posible y lo necesario. México, D.F.: Fondo de Cultura Económica. 
Martínez, A. T. (2007). Pierre Bourdieu: razones y lecciones de una práctica sociológica : del estructuralismo genético a la sociología reflexiva. Buenos Aires: Ediciones Manantial.

Munita, F. (2014a). El mediador escolar de lectura literaria. Un estudio del espacio de encuentro entre prácticas didácticas, sistemas de creencias y trayectorias personales de lectura. (Tesis de doctorado inédita). Universitat Autònoma de Barcelona, Barcelona.

Munita, F. (2014b). Trayectorias de lectura del profesorado en formación. Cultura y Educación/Culture and Education, 26(3), 448-475. https://doi.org/10.1080/11356405.2014.965449

Peroni, M. (2003). Historias de lectura. Trayectorias de vida y de lectura. México D.F.: Fondo de Cultura Económica.

Riessman, C. K. (2008). Narrative methods for the human sciences. Thousand Oaks, London, New Delhi, Singapore: Sage Publications, Inc.

Rockwell, E. (2001). La lectura como práctica cultural: conceptos para el estudio de los libros escolares. Educação e Pesquisa, 27(1), 11-26. https://doi.org/10.1590/s1517-97022001000100002

Rosenthal, G. (2004). Biographical research. En C. Seale, G. Gobo, J. F. Gubrium, \& D. Silverman (Eds.), Qualitative research practice: Concise paperback edition (pp. 48-64). Thousand Oaks, London, New Delhi: Sage Publications, Inc.

Shove, E. \& Pantzar, M. (2005). Consumers, producers and practices. Understanding the invention and reinvention of Nordic walking. Journal of consumer culture, 5(1), 43-64. https://doi. org/10.1177/1469540505049846

Silber, I. F. (2003). Pragmatic sociology as cultural sociology. Beyond repertory theory? European Journal of Social Theory, 6(4), 427-449. https://doi.org/10.1177/13684310030064004

Smith, B. \& Sparkes, A. C. (2008). Contrasting perspectives on narrating selves and identities: an invitation to dialogue. Qualitative Research, 8(1), 5-35. https://doi.org/10.1177/1468794107085221

Solé, I. (2009). Estrategias de lectura. Barcelona: Graó.

Taylor, D. (2015). El archivo y el repertorio. La memoria cultural performática en las Américas. Santiago de Chile: Ediciones Alberto Hurtado. 
Zavala, V. (2009). La literacidad o lo que la gente hace con la lectura y la escritura. En D. Cassany (Ed.), Para ser letrados (pp. 23-35). Barcelona: Paidós. 